\title{
High Blood Pressure Confirmed with 24-Hour Ambulatory Blood Pressure Monitoring in Tanzanian Adolescents Enrolling in a Cohort Study
}

\author{
Mussa Nsanya ( $\square$ kelvin.nsanya@gmail.com ) \\ Mwanza Intervention Trials Unit \\ Philip Ayieko \\ London School of Hygiene \& Tropical Medicine \\ Ramadhan Hashim \\ Mwanza Intervention Trials Unit \\ Ezekiel Mgema \\ Mwanza Intervention Trials Unit \\ Daniel Fitzgerald \\ Weill Cornell Medical College, New York

\section{Saidi Kapiga} \\ London School of Hygiene \& Tropical Medicine

\section{Robert Peck} \\ Weill Cornell Medical College, New York
}

\section{Research Article}

Keywords: sub-Saharan Africa, Tanzania, Adolescents, High Blood Pressure, White coat hypertension, Screening, 24-Hour Ambulatory Blood Pressure Monitoring (24-Hour ABPM)

Posted Date: December 15th, 2020

DOI: https://doi.org/10.21203/rs.3.rs-123312/v1

License: (c) (1) This work is licensed under a Creative Commons Attribution 4.0 International License. Read Full License

Version of Record: A version of this preprint was published at Scientific Reports on April 16th, 2021. See the published version at https://doi.org/10.1038/s41598-021-87996-0. 
High Blood Pressure Confirmed with 24-Hour Ambulatory Blood Pressure Monitoring in Tanzanian Adolescents Enrolling in a Cohort Study.

Mussa K. Nsanya ${ }^{1 *}$, Philip Ayieko ${ }^{1,2}$, Ramadhan Hashim ${ }^{1}$, Ezekiel Mgema ${ }^{1}$, Daniel Fitzgerald $^{3}$, Saidi Kapiga ${ }^{1,2}$, Robert N. Peck ${ }^{1,3}$

${ }^{1}$ Mwanza Intervention Trials Unit, National Institute for Medical Research, Mwanza, Tanzania

${ }^{2}$ London School of Hygiene and Tropical Medicine, London, UK

${ }^{3}$ Weill Cornell Medical College, New York, USA

${ }^{*}$ Corresponding Author: Correspondence concerning, and reprints request of this article should be addressed to Mussa K. Nsanya, e-mail: mussa.nsanya@mitu.or.tz. 


\section{ABSTRACT}

Prevalence estimates for high blood pressure (BP) among adolescents in sub-Saharan Africa vary widely and most studies have relied on attended automated office BP (AOBP) measurements. We aimed to estimate prevalence of high BP using unattended AOBP followed by 24-Hour ambulatory BP monitoring (24-Hour ABPM) for confirmation and to determine factors associated with confirmed high BP. Between April and August 2018, 500 adolescents aged 11 to 15 years were enrolled from three randomly selected public schools in Mwanza city (Tanzania) to participate in a 2-year prospective cohort study. We obtained three consecutive unattended AOBP measurements. Follow-up AOBP measurements were obtained in participants with high BP at initial measurements. Participants whose follow-up measurements remained high underwent 24-Hour ABPM. Of all 500 participants, $36.6 \%$ had high BP using first AOBP measurement; $25.6 \%$ using average of the second and third AOBP measurements and $10.2 \%$ using average of followup AOBP measurements and were eligible for 24-Hour ABPM. Only 13(2.6\%) had confirmed high BP and they had no unique distinguishing characteristic. White coat hypertension is common among adolescents in Africa. Cardiovascular health promotion in Africa can be done through school-based screening for high BP among adolescents using one unattended AOBP.

Keywords: sub-Saharan Africa; Tanzania; Adolescents; High Blood Pressure; White coat hypertension; Screening; 24-Hour Ambulatory Blood Pressure Monitoring (24-Hour ABPM) 


\section{INTRODUCTION}

High blood pressure (BP) and cardiovascular diseases (CVDs) are emerging in epidemic proportions among young adults in sub-Saharan Africa (SSA). In a population survey conducted in Tanzania and Uganda, the prevalence of high BP among young people ranged from $3 \%$ to $19 \%$ with males having higher burden than females ${ }^{1}$. In SSA, the rising prevalence of high BP has a disproportional impact on individuals, families, health systems and economies due to relatively young age when CVD related deaths occur ${ }^{2,3}$. In addition, primary health care facilities in SSA are poorly prepared for diagnosis and management of hypertension ${ }^{4-6}$ leaving few adolescents and young people screened for hypertension. This situation sets back efforts for cardiovascular health promotion in this population.

Prevalence estimates for high BP among adolescents and young people in SSA varies widely across studies ${ }^{7-13}$. This is partly due to differences in sampling methods and BP measurement procedures ${ }^{7,14}$. Additionally, in settings where childhood malnutrition is common, potential errors may arise from BP misclassifications in stunted subjects ${ }^{15}$. Attended office BP has remained the mainstay of BP measurement ${ }^{12}$ despite guidance on use of unattended office BP and 24-Hour ambulatory BP monitoring (24-Hour ABPM) as a reliable and accurate tool for confirming high BP which is more predictive of future cardiovascular disease ${ }^{16,17}$.There is scarcity of data on use of unattended AOBP and 24Hour ABPM among adolescents in sub-Saharan Africa ${ }^{18,19}$, raising concerns on the prevalence estimates for high BP among adolescents. 
Also, there are gender differences in distribution of risk factors to CVDs. A population survey among young adults living in urban setting in Tanzania found that compared to men, women have 4.3 higher odds of obesity and 3-folds greater odds for metabolic syndrome. In contrast, women had $50 \%$ lower odds for hypertension ${ }^{20}$. These differences have a physiological origin and may track back to childhood and adolescences ${ }^{21}$. However, in SSA cultural norms which define patterns of diet and physical activity may partly add to these differences ${ }^{22}$.

We therefore conducted a cross sectional analysis of data collected from 500 adolescents enrolling to a prospective cohort study of BP in SSA. We used unattended automated office BP (AOBP) measurements obtained on two different days followed by the gold standard 24-Hour ABPM measurement performed on adolescents with persistently high BP during follow up AOBP measurements (done in those with high BP during AOBP measurements obtained at day 1). Our overall objective was to ascertain the best strategy for screening of high BP and CVD risk factors in African adolescents. Our specific objectives were: 1) to determine the prevalence of confirmed high BP using the standard 24-Hour ABPM 2) to determine factors associated with high ABPM and 3) to describe traditional, endemic and ABPM risk factors for CVD and how these differ by gender.

\section{RESULTS}

\section{Enrollment summary}

Of the 952 randomly selected participants in three schools, 545 (57.3\%) obtained parents/guardians informed written consent for participation. And of those with parental consent, 500 (91.7\%) gave their written informed assent and were enrolled in the study. 
Reasons for not being enrolled were; declining assent 41 (7.5\%) and being considered ineligible $4(0.08 \%)$ due to short duration (less than 1 year) of residence in Mwanza city. AOBP measurements were available for all 500 enrolled participants. There were no systematic differences in age, sex and level of education between enrolled and declined participants.

Of all 500 enrolled participants, 51(10.2\%) were eligible for 24-Hour ABPM but we successful conducted the study in 45 (88.2\%). Reasons for not performing 24-Hour ABPM were: death (1), refusal (1) and moving out of Mwanza city (4). 24-Hour ABPM data from all 45 participants were available for analysis.

\section{Background characteristics}

In Table 1, we present the overall background characteristics of the 500 enrolled study participants by their gender groups. The mean age was $13.9(0.8)$ years. Majority of the participants were females (56.6\%); had lived in Mwanza city for more than 10 years $(85.2 \%)$; were in lower wealth index $(57.6 \%)$ and had their BP being measured for the first time in this study (91.2\%).

Of all participants, $62.2 \%$ and $79.9 \%$ never consumed fruits and vegetables respectively in a typical week. Similar proportion of participants reported less than the recommended levels of moderate and vigorous physical activity respectively. Of all study participants, $59 \%$ reported using an equivalent of 10 or more teaspoons of sugar in a typical day. Majority of the participants (80.8\%) had normal BMI, however about $70 \%$ of underweight participants were males and more than $80 \%$ of overweight or obese participants were females. In addition, $14 \%$ of all participants were stunted and majority of them (70\%) 
were males. In figure 1, we have summarized risk factors to cardiovascular disease which were statistically significant different between males and females.

Prevalence of kidney dysfunction (eGFR $\leq 60 \mathrm{mls} / \mathrm{min} / 1.73 \mathrm{~m}^{2}$ ) during enrollment was $10.4 \%$ (52). The burden was reduced by half, $5.4 \%$ (27) during repeat measurements done after six months. During enrollment, $6.6 \%$ had overt proteinuria and only $1 \%$ and $17.6 \%$ had sickle cell disease and trait respectively.

The 45 participants who underwent 24-Hour ABPM study had mean age of 14.0 (0.8) years, $56 \%$ were females and majority (71\%) were in lower wealth index. Additionally, $93 \%$ had normal BMI for their age, $11 \%$ were stunted and $16 \%$ had kidney dysfunction (eGFR below $60 \mathrm{mls} / \mathrm{min} / 1.73 \mathrm{~m}^{2}$ ).

\section{Prevalence of high AOBP and 24-Hour ABPM}

In Figure 2, we present the prevalence of high BP using the three methods used in this study. The first estimate is based on the first method in which the prevalence of high BP was $36.6 \%$ ( $95 \% \mathrm{Cl}: 32.5 \%$ to $40.9 \%$ ). The second estimate is based on the second $\underline{\text { method, }}$ in which the prevalence of high BP was $25.6 \%$ (95\% Cl: $21.8 \%$ to $29.7 \%$ ). The third estimate is based on the third method in which the prevalence of persistently high BP was $10.2 \%$ (95\% Cl: $7.7 \%$ to $13.2 \%)$. Importantly, all participants with normal BP using the first method $(63.4 \%)$ remained in the same BP classification during subsequent (second and third) AOBP measurements.

Of the 45 participants who underwent 24-Hour ABPM studies, 13 (28.9\%) had confirmed high BP using 24-Hour ABPM, of whom 12 had pre-hypertension and 1 had severe hypertension. The remaining $32(71.1 \%)$ had white coat hypertension. The 'true' overall 
prevalence estimate of high BP at enrollment was therefore (13/500), 2.6\% (95\%Cl, 1.4\% to $4.4 \%)$.

In Table 2, we present gender differences in distribution of 24-Hour ABPM characteristics. Males had significantly higher awake diastolic BP load $(p=0.009)$. In contrast, females had significantly higher ambulatory heart rate while awake $(p=0.0003)$ and while asleep $(p=0.0006)$

In supplementary Table, we compared socio-demographic, behavior and biological characteristics of participants with normal AOBP $(n=449)$ to those with white coat hypertension ( $n=32$ ) and those with confirmed high BP using 24-Hour ABPM ( $n=13)$. There was no statistically significant difference between the paired comparisons except for pulse rate. 


\section{DISCUSSION}

In this school-based cohort study of 500 Tanzanian adolescents from 3 different public schools in Mwanza city, Tanzania, we report results from baseline BP screening using three different methods. This study demonstrated that it is both acceptable and feasible to measure BP using a range of methods in the school settings. Although about $36 \%$ of study participants had high BP using the first BP measurement collected on the first day, less than 3\% were confirmed to have high BP using the 24-Hour ABPM reference standard procedure. Overall, more than two-third of participants had white coat hypertension and such participants could not be easily distinguished from those with confirmed hypertension based on their background characteristics. We also found that traditional, endemic and ABPM-related CVD risk factors were common and differed significantly by gender.

Using one AOBP measurement could provide a unique opportunity for school based hypertension screening and cardiovascular health promotion in countries like Tanzania. In this study, more than $90 \%$ of study participants never had their BP measured before the day of enrollment; a number we feel is representative of the general population of adolescents in Tanzania and sub-Saharan Africa at large where existing adolescent health programs have traditionally focused on sexual and reproductive health ${ }^{12}$. In addition, repeating $A O B P$ measurements only in those identified with high $B P$ during initial measurements saves time and has been recommended for adolescents ${ }^{23}$. It has been reported that more than $90 \%$ of adolescents aged $13-18$ years participating in the US National Health and Nutrition Examination Survey (NHANES) had their BP classification remaining the same in follow up measurements and $<3 \%$ had an increase in BP 
classification ${ }^{23,24}$. Similarly, in this study, all participants with normal BP using the first method (63.4\%) remained in the same BP classification during subsequent (second and third) BP measurements.

Our data suggest that white coat hypertension is extremely common in adolescents in Tanzania. This observation could partly be attributed to majority of the participants having their BP measured for the first time and anxiety to some of the study procedures such as physical examination and blood sampling or it could be related to imbalances in the autonomic nervous system with sympathetic overload. The high prevalence of white coat hypertension that we report from Tanzania is higher than estimates from adolescents in high-income countries ${ }^{25}$ and it underscores the importance of taking multiple AOBP measurements over multiple days followed by 24-Hour ABPM for accurate estimation of high BP. However, white coat hypertension may not be entirely benign.

Adults with white coat hypertension have consistently been shown to have higher incident hypertension and CVDs compared to those with normal office $\mathrm{BP}{ }^{26}$. Longitudinal studies of white coat effects among adolescents in Africa are needed.

Adolescents with confirmed high BP using the reference standard 24-Hour ABPM could not be easily separated from other participants based on socio-demographic, behavior and biological risk factors for high BP. In fact, none of the baseline background characteristics from Table 1 differed significantly between participants with confirmed high BP (using 24-Hour ABPM) versus those with normal AOBP or white coat hypertension (normal 24-Hour ABPM). This finding underscores the need to measure BP on all adolescents regardless of their apparent risk and to confirm high BP using 24-hour ABPM, as recommended by international guidelines ${ }^{16,17}$. Encouragingly, $90 \%$ (45/51) of eligible 
adolescents and their parents agreeing to participate in 24-Hour ABPM study indicates that it is also feasible to conduct 24-Hour ABPM in Tanzania and it may even be possible to do this in school-based health programs.

Traditional, endemic and ABPM risk factors for CVD are common among adolescents in Tanzania but differed from US and European adolescents. Although obesity was relatively rare, other traditional risk factors related to diet and physical activity were common. For example, $62.2 \%$ and $79.9 \%$ did not consume any serving of fruits and vegetables respectively in a typical week, which is far lower than the recommended 5 daily servings ${ }^{27}$. Since fruits and vegetables are high in potassium, low consumption could contribute directly to BP in Africa ${ }^{28}$. In addition, $5.4 \%$ of adolescent participants had kidney dysfunction (eGFR of $<60 \mathrm{mls} / \mathrm{min}$ ) at six months apart of repeated serum creatinine testing. We have previously reported that reduced kidney function is common in both children and young adults in Tanzania ${ }^{29,30}$, and is related to both neglected tropical disease, environmental exposures in mining communities and childhood undernutrition ${ }^{31}$. Therefore, reduced kidney function might represent an important endemic risk factor for early CVD in Tanzania. Importantly, one quarter of the adolescents with 24-Hour ABPM data had evidence of non-dipping, majority of them involving systolic BP and without gender differences. Nocturnal non-dipping with or without high BP is an established modifiable risk factor for cardiovascular diseases particularly among people of African origin $^{32}$ and has been associated with stroke and left ventricular hypertrophy ${ }^{33,34}$. Further studies are needed to determine the longitudinal trajectory of these risk factors and association with CVD in later adolescence and early adulthood. 
The distribution of CVD risk factors among adolescents in Tanzania differs significantly by gender. For instance, alcohol use, participation in vigorous physical activity, stunted growth and underweight were more common in males, whereas, females were more likely to be overweight or obese and report the recommended level of moderate and vigorous physical activity. Interestingly, females had relatively higher average pulse rate (which is a marker for lower physical fitness). Similar findings have been reported elsewhere in Africa ${ }^{8,13,22,35}$. Also, 24-Hour ABPM characteristics including heart rate and diastolic BP load varied significantly by gender with male adolescents generally having higher ABPM parameters than their female counterparts ${ }^{16}$. Similar findings have been previously reported ${ }^{36-38}$. While these differences are partly known to have their basis in hormonal and hemodynamic differences between males and females ${ }^{21}$, social and cultural norms which define gender roles could also be contributing to the observed differences in the Tanzanian context ${ }^{22,39}$.

Our study has strengths and limitations. One major strength of our study was the high rate of follow-up from initial BP measurement to repeat measurements to 24-hour ABPM. On the other hand, these are the limitations of this study; the relatively small sample size available for 24-Hour ABPM may have limited our statistical power to detect potential distinguishing features for those with high BP using 24-Hour ABPM. Also lack of ABPM data from participants with normal BP using unattended AOBP limited our assessment of 'masked hypertension' in this group of African adolescents. Lastly, the relatively low response of parents/guardians to provide consent for their children's participation in the study may have biased our findings. 
In conclusion, white coat hypertension is extremely common among adolescents in Tanzania. Adolescents with confirmed high BP using 24-Hour ABPM cannot be easily distinguished from those with white coat hypertension or normal clinic BP. Standard procedure for diagnosis of high BP in African adolescents should therefore include 24Hour ABPM, which appears to be acceptable and feasible in the Tanzanian context. School-based screening for high BP among adolescents with a single BP measurement would identify adolescents who need further BP evaluation and could also provide a platform for broader CVD health screening and education. Cardiovascular health education in Tanzanian schools should address traditional and endemic risk factors and should also provide gender-specific messages.

\section{METHODS}

\section{Study design, setting and sampling}

Between April and August 2018, we enrolled 500 secondary school students in a 2-year prospective cohort study in Mwanza city, which is Tanzania's second largest city located on the southern shores of Lake Victoria with an estimated population of about 1 million people and a blend of urban, semi-urban and rural communities. About $23 \%$ of the population in Tanzania is adolescents and the overall secondary school net enrollment is about $33 \%{ }^{40}$.

We initially prepared a list of secondary schools ( 20 public and 1 private) that enrolls both male and female students and are located within a radius of 5 kilometers from Mwanza city centre. From this list, three public schools were randomly selected to participate in the study. 
At each school, a de-identified list of students in the first two years of secondary

education with information on their date of birth was obtained. Using this list, about 300

students aged 11 to 15 years were selected using random sampling stratified by age to ensure proportional enrollment across the eligible age range.

Parents/guardians of selected students were invited to meet with the study team at the school to obtain information about the study and provide consent for their children to be enrolled in the study. Students whose parents/guardians did not attend the meeting at school were given reminder letters to take home. The letter provided detailed study and contact information and consent forms for the parents/guardians to sign if they wanted their children to join the study. For students with documented approval of parents/guardians, we sought their assent. Students who had lived in Mwanza city for less than one year were excluded due to concerns that they may move away from the city before the end of the study period.

\section{Data collection procedures}

A structured questionnaire adapted from the WHO STEPwise approach to noncommunicable diseases (NCDs) risk factors survey (STEPS instrument) ${ }^{41}$ was used. The questionnaire was translated into the local language (Kiswahili) and then independently back-translated to English for validation. We obtained information about sociodemographic characteristics and potential risk factors for high BP. We also collected information about parent/guardian's vital status, occupation and ownership of common household items which were used to generate categories of participant's wealth index. 
We collected data using handheld electronic devices (TECNO ${ }^{\circledR}$ Android Tablets)

programmed to check for accuracy and consistency of entered data.

After the interviews, we conducted physical examination to collect anthropometric measurements. Height was measured in an upright standing posture on bare foot and without head ornament, using a portable stadiometer (Seca ${ }^{\circledR} 213$, Seca GmbH \& co. KG., Hamburg, Germany) at a precision of $0.1 \mathrm{~cm}$. Waist and hip circumference were measured over a single layer of clothing using a tape measure (Seca ${ }^{\circledR} 201$, Seca GmbH \& co. KG, Hamburg, Germany) at a precision of $0.1 \mathrm{~cm}$. Weight was measured with a participant in an upright standing posture, in light clothing and on bare foot, using a digital weight scale (Seca ${ }^{\circledR} 876$ flat scale, Seca GmbH \&co. KG., Hamburg Germany) at a precision of $0.1 \mathrm{~kg}$. The weight and height measurements were used to calculate the body mass index (BMI) which was categorized into underweight, normal weight, overweight or obesity using the WHO's BMI for age Z-scores ${ }^{42}$.

We obtained AOBP using a validated monitor (OMRON ${ }^{\circledR}$ Model BP791IT - HEM-7222-ITZ, Omron Health care Inc., Lake Forest, Illinois - USA) while participants were seated in an upright position with an appropriate cuff size as determined from the participant's right mid-upper arm circumference (MUAC). The BP measurement procedure was fully explained, and after resting for at least 5 minutes in a booth, the participant started the BP machine and three consecutive measurements were automatically obtained at an interval of 1 minute and precision of $0.1 \mathrm{mmHg}$. The average of the last two AOBP measurements obtained on Day 1 were used to screen for high BP using the screening algorithm of the 2017 American standard guideline ${ }^{17}$. Participants aged $\geq 13$ years and whose AOBP measurements were $\geq 120 / 80 \mathrm{mmHg}$, or those aged $<13$ years and whose 
AOBP was $\geq 90^{\text {th }}$ percentile for their age, height and sex screened positive for high BP and were eligible for follow-up AOBP measurements one week later (Day 2) using similar procedure. The average of last two AOBP measurements obtained on Day 2 was used to obtain final BP percentiles using BP reference tables which takes into account participant's age, sex and height [also available in a Statistical Analysis System (SAS) code] in the guideline ${ }^{17}$. Participants with $\geq 90^{\text {th }}$ percentiles for systolic and/or diastolic AOBP were regarded as having persistently high BP and were eligible for 24-Hour ABPM. After physical examination and $A O B P$, we collected $5 \mathrm{~mL}$ of venous whole blood for malaria, hemoglobin electrophoresis, and serum creatinine tests. While in the field, we used two drops of blood to test for malaria using rapid diagnostic test (ACCESS BIO, 65 Clyde Road, New Jersey, USA), and five drops of blood for preparing samples of dried blood spot (Whatman 903 filter paper). The remaining blood sample was transported to the central laboratory at the National Institute for Medical Research (NIMR - Mwanza) for further processing and testing. We also collected about $40 \mathrm{~mL}$ of urine sample for urine tests including $10 \mathrm{~mL}$ which was used for urine strip analysis (ACON laboratories Inc, San Diego, California - USA) at the field. We communicated abnormal results to the respective participants in presence of their parents/guardians. Additionally, participants with sickle cell trait or disease were counseled on what the results meant, what to expect and what they were supposed to do to modify the course of their disease as well as on the importance of screening for the disease before marriage.

\section{Laboratory procedures}

At a central NIMR laboratory, about $30 \mathrm{~mL}$ of the remaining urine was centrifuged and aliquoted in two cryotubes $\left(1.8 \mathrm{~mL}\right.$ each) and stored at $-80^{\circ} \mathrm{C}$ for future testing. 
The dried blood spot samples were kept overnight in a clean environment to air-dry. They were then sealed in labeled bags and stored at a temperature of $-80^{\circ} \mathrm{C}$. After six months, we performed hemoglobin electrophoresis to characterize participants' hemoglobin as normal, sickle cell trait or sickle cell disease hemoglobin (Multiphor II Electrophores is Unit, 751 84, Uppsala Sweden).

The remaining whole venous blood was centrifuged to separate serum and two $1.8 \mathrm{~mL}$ aliquots were prepared and stored at $-80^{\circ} \mathrm{C}$ for future testing. One of the stored aliquots was used for serum creatinine testing (A25 Biosystems SA. Costa Brava, 30. Barcelona, Spain) whose results were used to calculate Estimated Glomerular Filtration Rate (eGFR) based on the modified Schwartz equation ${ }^{43}$. Participants with eGFR below 60 $\mathrm{mls} / \mathrm{min} / 1.73 \mathrm{~m}^{2}$ had their blood collected again after 6 months for a repeat test to confirm the low eGFR.

\section{4-Hour Ambulatory Blood Pressure Monitoring (24-Hour ABPM) data collection} procedure

The 24-Hour ABPM studies were conducted by a trained study nurse using a validated monitor (Oscar 2, SunTech Medical) during routine school days ${ }^{16}$. The monitor was programmed using AccuWin Pro 4 software to obtain BP measurements every 15 minutes for 24 hours $^{16}$. Mid-upper arm circumference was used to determine the appropriately sized cuff, which was placed on a non-dominant arm and connected to the battery powered ABPM monitor using rubber tubing. While at school during routine school days, we started the monitor and confirmed that it was functioning by observing two consecutive readings before allowing the participant to leave. After 24 hours, we removed the ABPM monitor and downloaded the data. Sleep and wake times were 
defined using participant's diary as recommended for adolescents ${ }^{44}$. Height, gender and age were entered to obtain the participant's BP category. Times when participants were active or distressed were noted and used to interpret results. Pre-specified 24-Hour ABPM characteristic were extracted to data collection forms for double data entry using Open Clinica version 3.1.4. Participants with high 24-Hour ABPM were counseled on diet and lifestyle changes and their BP were followed up with during the study (if they had pre-hypertension) OR they were referred to specialist care (if they had hypertension) ${ }^{17}$.

\section{Statistical analysis procedures}

We had three AOBP measurements obtained on Day 1 (for all participants) and additional three follow-up AOBP measurements obtained on Day 2 (only for those with high BP on Day 1). We used three methods to estimate the prevalence of high AOBP and their corresponding 95\% confidence intervals using interval estimate on for binomial proportion. Two prevalence estimates for high BP were calculated using AOBP measurements obtained on Day 1 while the third prevalence estimate was based on AOBP measurements obtained during the follow-up visit on Day 2. The first method, used the first AOBP measurement (in a series of three) obtained on Day 1 to screen for high BP using the 'screening algorithm' of the 2017 American standard guideline ${ }^{17}$. Participants with $B P \geq 120 / 80 \mathrm{mmHg}$ if aged $\geq 13$ years or $B P \geq 90^{\text {th }}$ percentile if aged $<13$ years were regarded as having high $\mathrm{BP}$. The second method used the average of the second and third AOBP measurements (in a series of three) obtained on Day 1 to screen the proportion of participants with high BP using the screening algorithm (BP $\geq 120 / 80 \mathrm{mmHg}$ if aged $\geq 13$ years or $\mathrm{BP} \geq 90^{\text {th }}$ percentile if aged $<13$ years). The third method only involved participants with high BP using the second method, and for these we used the average of 
their second and third follow-up AOBP measurements obtained on Day 2 to obtain their 'final' BP using the BP percentile tables ${ }^{17}$. Participants whose systolic and/or diastolic BP $\geq$ $90^{\text {th }}$ percentiles for their age, sex and height were regarded as having persistently high BP underwent 24-Hour ABPM. We then categorized 24-hour average BP as either normal BP OR pre-hypertension OR hypertension OR severe hypertension, depending on participant's age, sex and height as per the American Heart Association definitions ${ }^{16}$. Participants whose average 24-Hour ABPM was categorized as pre-hypertension, hypertension or severe hypertension were regarded as having high 24-Hour ABPM while those with normal 24-Hour ABPM were regarded as having white coat hypertension. Participants' demographic, biological and behavioral characteristics were summarized for males and females separately using median [IQR] for continuous variables and proportions for categorical variables. To test for statistical significant differences between males and females, Student's T-test (continuous variable) and Chi-squared test (for categorical variable) were used.

To assess for factors associated with high 24-Hour ABPM, we compared background characteristics of participants with normal AOBP to those with high 24-Hour ABPM and those with white coat hypertension. Student's T test (continuous variable) and Fisher's exact test (categorical variable) were used to test for statistical significant differences between the groups. Similar procedures were used to asses for differences in 24-Hour ABPM characteristics between males and females. In order to adjust for potential type 1 error resulting from multiple comparisons, we only reported ABPM characteristics with $p$ value $<0.01$ as statistically significant. Statistical analysis was performed using Stata IC version 14 (StataCorp, College Station, Texas, USA). 


\section{Ethical considerations}

The study was approved by the ethics committee of the Tanzania National Institute for Medical Research (NIMR/HQ/R.8a/Vol.IX/2452) and the Weill Cornell Medical College Institutional Review Board (\#1612017830). Informed written consent from parents or guardians was obtained before participants were approached for their informed written assent. To ensure confidentiality, unique study identity numbers were used. All methods were performed in accordance with the relevant guidelines and regulations.

\section{References}

1. Kavishe, B. et al. High prevalence of hypertension and of risk factors for noncommunicable diseases ( NCDs ): a population based cross-sectional survey of NCDS and HIV infection in Northwestern Tanzania and Southern Uganda. BMC Med. 1-21 (2015) doi:10.1186/s12916-015-0357-9.

2. Gersh, B. J., Sliwa, K., Mayosi, B. M. \& Yusuf, S. Novel therapeutic concepts: The epidemic of cardiovascular disease in the developing world: Global implications. Eur. Heart J. 31, 642-648 (2010).

3. Peck, R. et al. Hypertension related diseases as a common cause of hospital mortality in Tanzania. A 3 year prospective study. J Hypertens 31, 1806-1811 (2014).

4. Bintabara, D. \& Mpondo, B. C. T. Preparedness of lower-level health facilities and the associated factors for the outpatient primary care of hypertension: Evidence from Tanzanian national survey. PLoS One 13, 1-14 (2018).

5. Katende, D. et al. Readiness of Ugandan health services for the management of outpatients with chronic diseases. Trop. Med. Int. Heal. 20, 1385-1395 (2015).

6. Peck, R. et al. Preparedness of Tanzanian heal th facilities for outpatient primary care of hypertension and diabetes : a cross-sectional. Lancet Glob. Heal. 2, e285e292 (2014).

7. Nsanya, M. K. et al. Prevalence of high blood pressure and associated factors among adolescents and young people in Tanzania and Uganda. J. Clin. Hypertens. 21, 470-478 (2019).

8. Ujunwa FA, Ikefuna AN, Nwokocha AR \& Chinawa JM. Hypertension and prehypertension among adolescents in secondary schools in Enugu, South East Nigeria. Ital. J. Pediatr. 1-6 (2013).

9. Nkeh-Chungag, B. N., Sekokotla, A. M., Sewani-Rusike, C., Namugowa, A. \& Iputo, J. E. Prevalence of hypertension and Pre-Hypertension in 13-17 year old adolescents living in Mthatha-South Africa: A cross-sectional study. Cent. Eur. J. Public Health 23, 59-64 (2015). 
10. Kidy, F. et al. Blood pressure in primary school children in Uganda: A cross-sectional survey. BMC Public Health 14, 1-9 (2014).

11. Jobe, M., Agbla, S. C., Prentice, A. M. \& Hennig, B. J. High blood pressure and associated risk factors as indicator of preclinical hypertension in rural West Africa: A focus on children and adolescents in The Gambia. Med. (United States) 96, (2017).

12. Noubiap, J. J. et al. Prevalence of elevated blood pressure in children and adolescents in Africa: a systematic review and meta-analysis. Lancet Public Heal. 2, e375-e386 (2017).

13. Ellenga Mbolla, B. F. et al. Prehypertension and hypertension among school children in brazzaville, congo. Int. J. Hypertens. 2014, 1-6 (2014).

14. de Oliveira, L. M. F. T. et al. The number of visits and blood pressure measurements influence the prevalence of high blood pressure in adolescents. J. Am. Soc. Hypertens. 11, 343-349 (2017).

15. Parker, E. D. et al. Potential Misclassification of Blood Pressure Status in Children and Adolescents with Short or Tall Stature. Am. J. Epidemiol. 183, 79-83 (2016).

16. Flynn, J. T. et al. Update: Ambulatory Blood Pressure Monitoring in Children and Adolescents. A scientific Statement from the American Heart Association. Hypertension 63, 1116-1135 (2014).

17. Flynn, J. T. \& Falkner, B. E. New clinical practice guideline for the management of high blood pressure in children and adolescents. Hypertension 70, 683-686 (2017).

18. Etyang, A. O. et al. Blood Pressure and Arterial Stiffness in Kenyan Adolescents with the Sickle Cell Trait. Am. J. Epidemiol. 187, 199-205 (2018).

19. Quinn, A. K. et al. Ambulatory monitoring demonstrates an acute association between cookstove- related carbon monoxide and blood pressure in a Ghanaian cohort. Environ. Heal. 1-14 (2017) doi:10.1186/s12940-017-0282-9.

20. Njelekela, M. A. et al. Gender-related differences in the prevalence of cardiovascular disease risk factors and their correlates in urban Tanzania. BMC Cardiovasc. Disord. 9, 1-8(2009).

21. Syme, C. et al. Sex Differences in Blood Pressure and Its Relationship to Body Composition and Metabolism in Adolescence. Arch Pediatr Adolesc Med 163, 818825 (2009).

22. Darling, A. M. et al. Gender differences in nutritional status, diet and physical activity among adolescents in eight countries in sub-Saharan Africa. Trop. Med. Int. Heal. 25, 33-43 (2020).

23. Lewis, M. N., Shatat, I. F. \& Phillips, S. M. Screening for hypertension in children and adolescents: Methodology and current practice recommendations. Front. Pediatr. 5, 1-5 (2017).

24. Becton, L. J., Egan, B. M., Hailpern, S. M. \& Shatat, I. F. Blood pressure reclassification in adolescents based on repeat clinic blood pressure measurements. J. Clin. Hypertens. 15, 717-722 (2013).

25. Sorof, J. M. \& Portman, R. J. White coat hypertension in children with elevated casual blood pressure. J Pediatr 137, 493-497 (2000).

26. Hanevold, C. D., Miyashita, Y., Faino, A. V. \& Flynn, J. T. Changes in Ambulatory Blood Pressure Phenotype over Time in Children and Adolescents with Elevated Blood Pressures. J. Pediatr. 216, 37-43.e2 (2020). 
27. WHO. Fruit and Vegetable Daily Requirement. http://www.who.int/dietphysicalactivity/fruit/en/.

28. Berhame, Y. et al. The age of opportunity: Prevalence of key risk factors among adolescents 10 - 19 years of age in nine communities in sub-Saharan Africa. Trop. Med. Int. Heal. 25, 15-32 (2020).

29. Kayange, N. M. et al. The Influence of HIV and Schistosomiasis on Renal Function: A Cross-sectional Study among Children at a Hospital in Tanzania. PLoS Negl. Trop. Dis. 9, 1-13 (2015).

30. Peck, R. et al. Decreased renal function and associated factors in cities, towns and rural areas of Tanzania: A community-based population survey. Trop. Med. Int. Heal. 21, 393-404 (2016).

31. Kayange, N. M. et al. Systematic review Systematic review Tasks. 313, 163-172 (2015).

32. Mezue, K. et al. Related Organ Damage $N$ octurnal $N$ on - dipping B lood P ressure $P$ rofile in $\mathrm{B}$ lack $\mathrm{N}$ ormotensives I s A ssociated with $\mathrm{C}$ ardiac $\mathrm{T}$ arget $\mathrm{O}$ rgan $\mathrm{D}$ amage. 26, 279-284 (2016).

33. Wilson, D. K., Sica, D. A. \& Miller, S. B. Effects of Potassium on Blood Pressure in Salt-Sensitive and Salt-Resistant Black Adolescents. Hypertension 34, 181-186 (1999).

34. O'Brien, E., James, S. \& Kevin, O. Dippers and non-dippers. Lancet 332, 397 ( 1988).

35. Nkeh-Chungag, B. N., Sekokotla, A. M., Sewani-Rusike, C., Namugowa, A. \& I puto, J. E. Prevalence of hypertension and Pre-Hypertension in 13-17 year old adolescents living in Mthatha-South Africa: A cross-sectional study. Cent. Eur. J. Public Health 23, 59-64 (2015).

36. Urbina, E. et al. Ambulatory blood pressure monitoring in children and adolescents: Recommendations for standard assessment: A scientific statement from the american heart association atherosclerosis, hypertension, and obesity in youth committee of the council on cardiovas. Hypertension 52, 433-451 (2008).

37. Harshfield, G. A. et al. Race and Gender Influence Ambulatory Blood Pressure Patterns of Adolescents. Hypertension 14, 598-603 (1989).

38. Kotsis, V. et al. Impact of gender on 24-h ambulatory blood pressure and target organ damage. J. Hum. Hypertens. 20, 658-665 (2006).

39. Benkeser, R. M., Biritwum, R. \& Hill, A. G. Prevalence of overweight and obesity and perception of healthy and desirable body size in urban, Ghanaian women. Ghana Med. J. 46, 66-75 (2012).

40. NBS. Tanzania Demographic and Health Survey, 2015/2016 - National Bureau of Statistics.

41. WHO. NCDs Surveillance STEPS Instrument. https://www.who.int/ncds/surveillance/steps/instrument/en/ (2005).

42. WHO. International Growth Reference. BMI for Age https://www.who.int/growthref/who2007_bmi_for_age/en/ (2007).

43. Zachwieja, K. et al. Modification of the Schwartz equations for children increases their accuracy at eGFR $>60 \mathrm{~mL} / \mathrm{min} / 1.73 \mathrm{~m} 2$. Ren. Fail. 38, 787-798 (2016).

44. Jones, H. E. \& Sinha, M. D. The definition of daytime and nighttime influences the interpretation of ABPM in children. Pediatr. Nephrol. 26, 775-781 (2011). 


\section{Acknowledgements}

The study team is grateful for the support of school board, administrators, and parents/guardians at the study secondary schools (Nyamanoro, Nyakabungo, and Pamba) and the adolescents who contributed to this research. We also wish to acknowledge the contributions made by our data collection staff (Amina Ally, Mariana Mathias, Jonathan Mbelwa and Simon Sichalwe).

\section{Author contributions}

All authors were involved in the study design, data collection, data management and interpretation of results. Data analysis was performed by MKN and PA. This submission was drafted by MKN and all authors were involved in editing and reviewing the manuscript. All authors approved the final version of this submission.

Source of Funding: This study was funded by a grant from the US National Institute of Health - Fogarty International Centre (NIH - FIC), Award Number [(5 R25 TW009337-05 (VUMC 58376)]. We also acknowledge support from the Centre for Global Health at the Weill Cornell Medicine.

Competing interests: The authors have no conflicts of interests to declare. 
Table1: Bas eline background characteristics of adol escents enrolled in a 2-year cohort study in Mwanza City, Tanzania ( $\mathrm{N}=500)$.

\begin{tabular}{|c|c|c|c|c|}
\hline Characteristic & $\begin{array}{c}\text { Male \& } \\
\text { Female } \\
\mathrm{N}=500 \\
\mathrm{n}(\%)\end{array}$ & $\begin{array}{c}\text { Male } \\
\mathrm{N}=217 \\
\mathrm{n}(\%)\end{array}$ & $\begin{array}{c}\text { Female } \\
\mathrm{N}=283 \\
\mathrm{n}(\%)\end{array}$ & $P$ - value \\
\hline \multicolumn{5}{|l|}{ Socio-demographic and Economic Factors } \\
\hline Age (years) ${ }^{1}$ & $13.9(0.8)$ & $14.1(0.8)$ & $13.8(0.8)$ & 0.0010 \\
\hline \multicolumn{4}{|l|}{ Duration of residence in Mwanza City } & $(0.0020)$ \\
\hline \multicolumn{4}{|l|}{ Wealth Index Group ${ }^{2}$} & $(0.20)$ \\
\hline Middle/Upper & $212(42.4)$ & $85(39.2)$ & $127(44.9)$ & \\
\hline Measured BP for the first time & $456(91.2)$ & $197(90.8)$ & $259(91.5)$ & 0.77 \\
\hline \multicolumn{5}{|c|}{ Behavior Factors } \\
\hline Ever smoked tobacco & $5(1.0)$ & $3(1.3)$ & $2(0.7)$ & 0.45 \\
\hline Ever drunk Alcohol & $46(9.2)$ & $31(14.3)$ & $15(5.3)$ & 0.0010 \\
\hline \multicolumn{5}{|l|}{ Fruits servings consumed per day } \\
\hline 0 & $311(62.2)$ & $137(63.1)$ & $174(61.5)$ & \\
\hline 1 & $111(22.2)$ & $38(17.5)$ & $73(25.8)$ & $(0.062)$ \\
\hline $2-4$ & $61(12.2)$ & $33(15.2)$ & $28(9.9)$ & \\
\hline$\geq 5$ & $17(3.4)$ & $9(4.2)$ & $8(2.8)$ & \\
\hline \multicolumn{5}{|l|}{ Vegetables servings consumed per day } \\
\hline 0 & $398(79.9)$ & $179(82.5)$ & $219(77.4)$ & \\
\hline 1 & $87(17.4)$ & $32(14.8)$ & $55(19.4)$ & $(0.36)$ \\
\hline$\geq 2$ & $15(3.0)$ & $6(2.8)$ & $9(3.2)$ & \\
\hline \multicolumn{5}{|l|}{ Teaspoons of sugar per day } \\
\hline$<10$ & $203(40.6)$ & $90(41.5)$ & $113(39.9)$ & $(0.73)$ \\
\hline$\geq 10$ & $297(59.4)$ & $127(58.5)$ & $170(60.1)$ & \\
\hline $\begin{array}{l}\text { Minutes spent in moderate physical } \\
\text { activity per day }\end{array}$ & & & & $(0.55)$ \\
\hline$\geq 60$ & $194(38.8)$ & $81(37.4)$ & $113(39.9)$ & \\
\hline $\begin{array}{l}\text { Days spent in vigorous physical } \\
\text { activity per week }\end{array}$ & & & & $(<0.0001)$ \\
\hline$\geq 3$ & $133(26.6)$ & $25(11.5)$ & $108(38.2)$ & \\
\hline \multicolumn{5}{|c|}{ Biological Factors } \\
\hline \multicolumn{5}{|l|}{ Body Mass Index (BMI) } \\
\hline Underweight & $57(11.5)$ & $40(18.4)$ & $17(6.0)$ & \\
\hline Normal & $405(80.8)$ & $170(78.3)$ & $235(83.0)$ & $(<0.0001)$ \\
\hline Overweight/Obesity & $38(7.7)$ & $7(3.2)$ & $31(11.0)$ & \\
\hline \multicolumn{5}{|l|}{ Pulse rate (beats/minute) } \\
\hline$<60$ & $25(5.0)$ & $23(10.6)$ & $2(0.7)$ & \\
\hline$\geq 60$ to $<90$ & $364(72.8)$ & $167(77.0)$ & $197(69.6)$ & $(<0.0001)$ \\
\hline$\geq 90$ & $111(22.2)$ & $27(12.4)$ & $84(29.7)$ & \\
\hline $\begin{array}{l}\text { Stunted growth }^{3} \\
\text { eGFR }^{4}\end{array}$ & $70(14.0)$ & $49(22.6)$ & $21(7.4)$ & $<0.0001$ \\
\hline Normal $\left(\geq 60 \mathrm{mls} / \mathrm{min} / 1.73 \mathrm{~m}^{2}\right)$ & $448(89.6)$ & $192(88.5)$ & $256(90.5)$ & $(0.42)$ \\
\hline $\begin{array}{l}\text { Renal dys function } \\
\left(<60 \mathrm{mls} / \mathrm{min} / 1.73 \mathrm{~m}^{2}\right)\end{array}$ & $52(10.4)$ & $25(11.5)$ & $27(9.5)$ & \\
\hline \multicolumn{5}{|c|}{${ }^{1}$ Mean (SD) } \\
\hline \multicolumn{5}{|c|}{$\begin{array}{l}{ }^{2} \text { Wealth Index: an aggregate variable generated by combining data on parents/guardian's vital status, } \\
\text { occupation and ownership of common household items. } \\
{ }^{3} \text { Stunted growth defined according to WHO - height for age below }-2 \text { standard deviation (SD) } \\
4 \text { Estimated Glomerular Filtration Rate (calculated using the modified Schwartz equation). } \\
\text { () - Overall p value }\end{array}$} \\
\hline
\end{tabular}


Table 2: Distribution of ambulatory BP characteristic by gender among secondary school adolescents in Mwanza City, Tanzania (N=45).

\begin{tabular}{|c|c|c|c|c|}
\hline Ambulatory BP Characteristic & $\begin{array}{l}\text { Males and } \\
\text { Females } \\
(\mathrm{N}=45) \\
\mathrm{N}(\%) \text { or } \\
\text { Mean (SD) }\end{array}$ & $\begin{array}{l}\text { Males } \\
(\mathbf{N}=\mathbf{2 0}) \\
\mathrm{N}(\%) \text { or } \\
\text { Mean (SD) }\end{array}$ & $\begin{array}{c}\text { Females } \\
(\mathrm{N}=\mathbf{2 5}) \\
\mathrm{N}(\%) \text { or } \\
\text { Mean (SD) }\end{array}$ & $\begin{array}{l}{ }^{*} P \\
\text { value }\end{array}$ \\
\hline $\begin{array}{l}\text { Confirmed high BP } \\
\text { ( } 24 \text { hour ABPM) }\end{array}$ & $13(29.0 \%)$ & $5(25.0 \%)$ & $8(32.0 \%)$ & NS \\
\hline $\begin{array}{l}\text { Average office systolic BP } \\
\text { (mmHg) }\end{array}$ & $125.9(7.4)$ & $127.4(7.5)$ & $124.7(7.2)$ & NS \\
\hline $\begin{array}{l}\text { Average office diastolic BP } \\
(\mathrm{mmHg})\end{array}$ & $76.8(6.7)$ & $76.2(6.1)$ & $77.3(7.3)$ & NS \\
\hline Average office heart rate (bpm) & 80.9 (16.9) & $74.0(13.0)$ & $86.4(17.9)$ & 0.01 \\
\hline 24-hour systolic ABPM (mmHg) & $111.6(6.7)$ & $113.7(8.2)$ & $110.0(4.7)$ & NS \\
\hline 24-hour diastolic ABPM ( $\mathrm{mmHg}$ ) & $64.8(4.5)$ & $64.8(5.2)$ & $64.9(3.8)$ & NS \\
\hline Asleep systolic ABPM (mmHg) & $102.8(7.4)$ & $104.9(9.2)$ & $101.2(5.3)$ & NS \\
\hline Asleep diastolic ABPM (mmHg) & $55.2(4.8)$ & $54.9(5.6)$ & $55.4(4.1)$ & NS \\
\hline Awake systolic ABPM (mmHg) & $117.3(6.8)$ & $119.5(7.9)$ & $115.6(5.4)$ & NS \\
\hline Awake diastolic ABPM (mmHg) & $70.6(5.9)$ & $70.4(7.3)$ & $70.8(4.5)$ & NS \\
\hline Awake ABPM heart rate (bpm) & $84.6(12.3)$ & $78.0(11.0)$ & $90.0(10.8)$ & 0.001 \\
\hline Asleep ABPM heart rate (bpm) & $68.3(10.7)$ & $62.8(10.6)$ & $72.7(8.6)$ & 0.001 \\
\hline Heart rate non-dipper $(<10 \%)$ & $2(4.4)$ & $1(5.0)$ & $1(4.0)$ & 0.01 \\
\hline Systolic BP dip (\%) & $12.4(4.0)$ & $12.3(4.6)$ & $12.5(3.6)$ & NS \\
\hline Diastolic BP dip (\%) & $22.3(5.7)$ & $23.2(6.3)$ & $21.5(5.2)$ & NS \\
\hline Systolic BP non-dipper (<10\%) & $11(24.4 \%)$ & $5(25.0 \%)$ & $6(24.0 \%)$ & NS \\
\hline Diastolic BP non-dipper (<10\%) & $1(2.2 \%)$ & $0(0.0 \%)$ & $1(4.0 \%)$ & NS \\
\hline $\begin{array}{l}\text { Combined BP non-dipper } \\
(<10 \%)\end{array}$ & $11(24.4 \%)$ & $5(25.0 \%)$ & $6(24.0 \%)$ & NS \\
\hline 24-hour systolic BP load (\%) & $12.5(11.0)$ & $13.5(13.5)$ & $11.7(8.6)$ & NS \\
\hline 24-hour diastolic BP load (\%) & $13.3(10.2)$ & $16.6(12.9)$ & $10.7(6.5)$ & NS \\
\hline Awake systolic BP load (\%) & $14.6(11.0)$ & $14.7(12.5)$ & $14.5(9.8)$ & NS \\
\hline Awake diastolic BP load (\%) & $15.8(11.9)$ & $20.4(14.7)$ & $12.1(7.6)$ & 0.01 \\
\hline Asleep systolic BP load (\%) & $9.5(14.0)$ & $12.1(18.5)$ & $7.4(8.8)$ & NS \\
\hline Asleep diastolic BP load (\%) & $9.7(11.3)$ & $11.7(15.0)$ & $8.2(7.2)$ & NS \\
\hline Morning BP surge (mmHg) & $21.3(13.2)$ & $20.6(12.2)$ & $21.8(14.1)$ & NS \\
\hline $\begin{array}{l}\text { Ambulatory Arterial Stiffness } \\
\text { Index (AASI) }\end{array}$ & $0.25(0.12)$ & $0.24(0.13)$ & $0.26(0.12)$ & NS \\
\hline
\end{tabular}




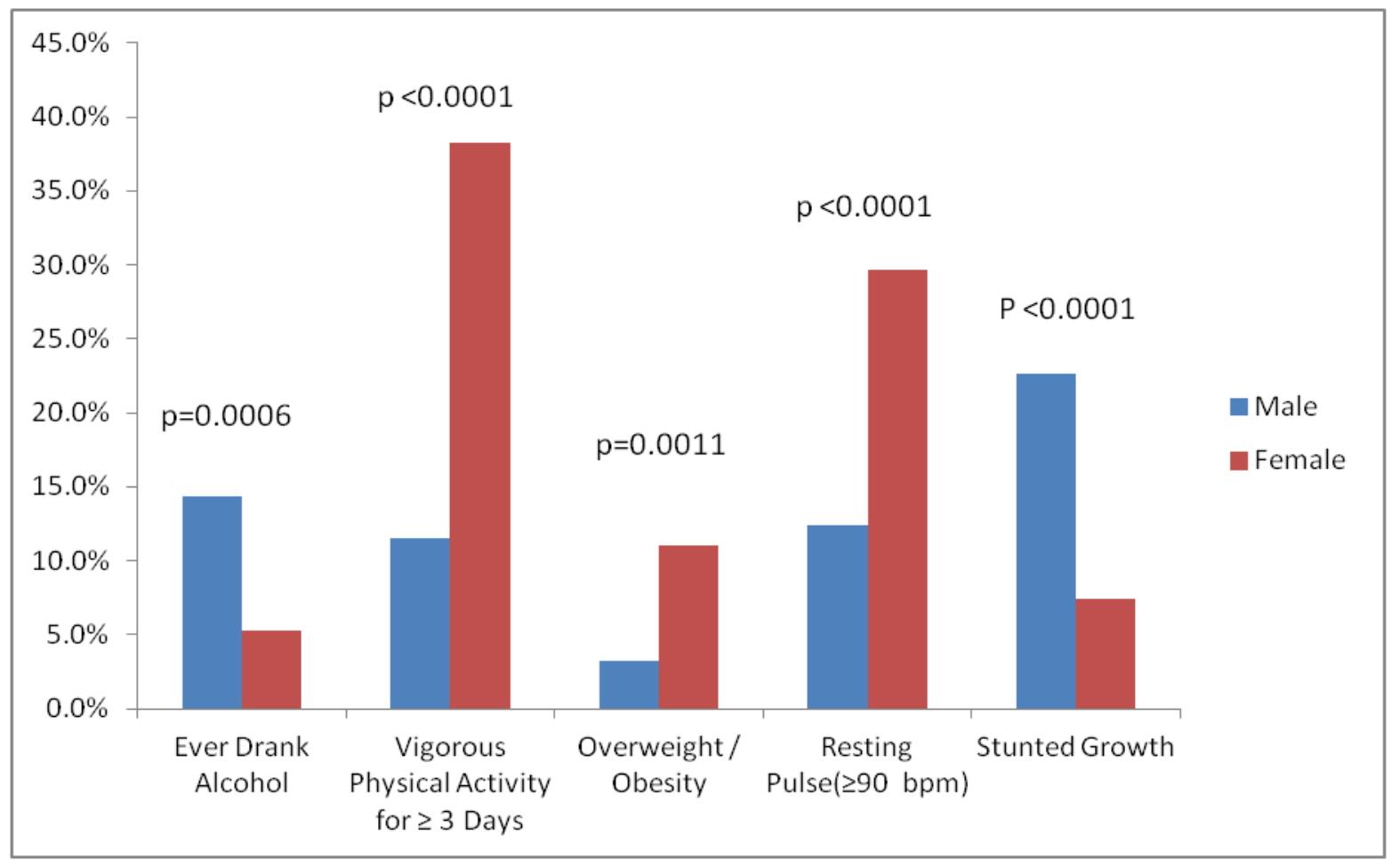

Figure 1: Bar graph showing risk factors for cardiovascular disease which were statistically significant different between males $(n=217)$ and females $(n=283)$ participating in adolescents' cohort study 


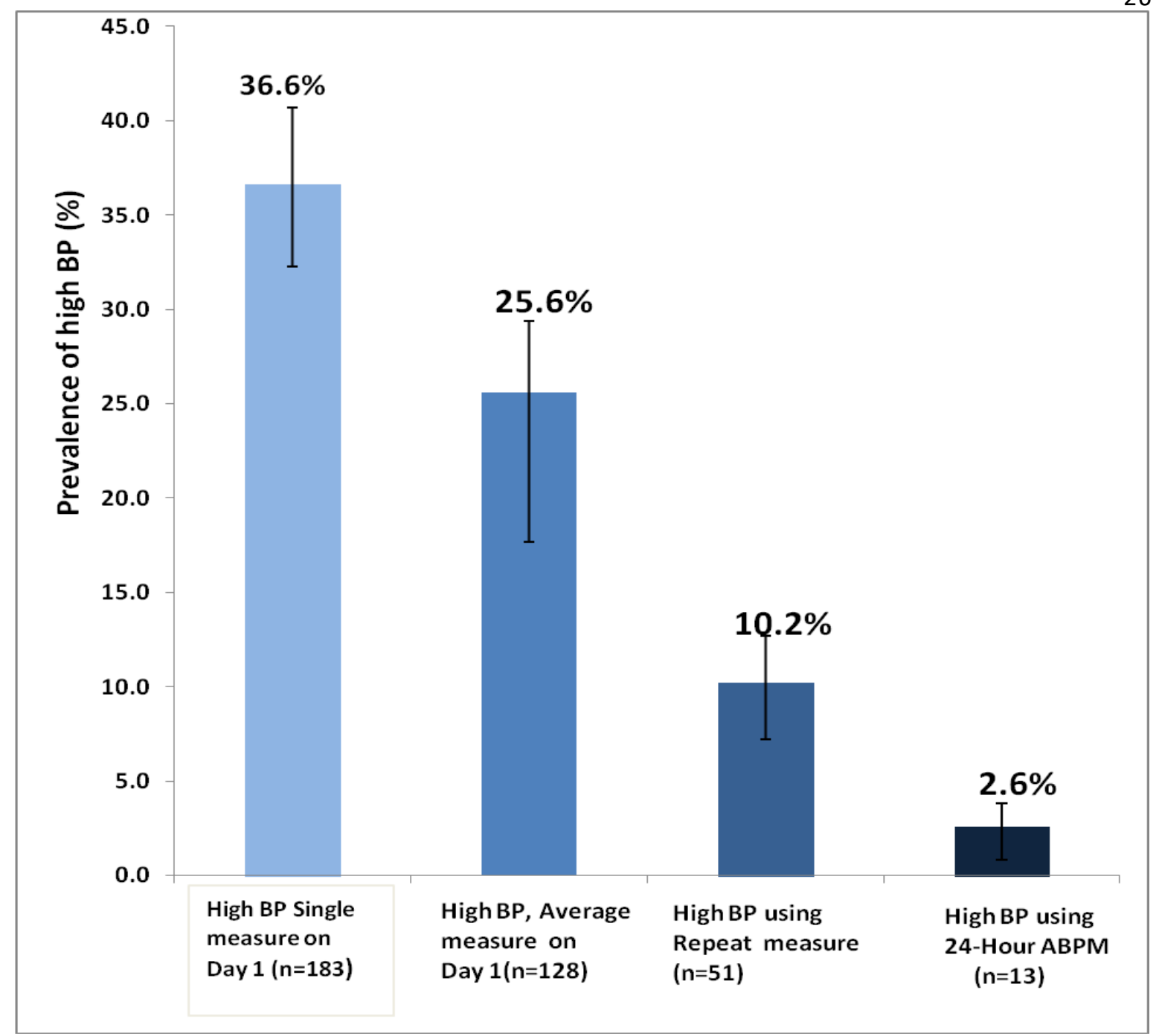

Figure 2: Prevalence of high BP with their corresponding estimation methods among adolescents enrolling in adolescents' cohort study $(\mathrm{N}=500)$. 
Figures

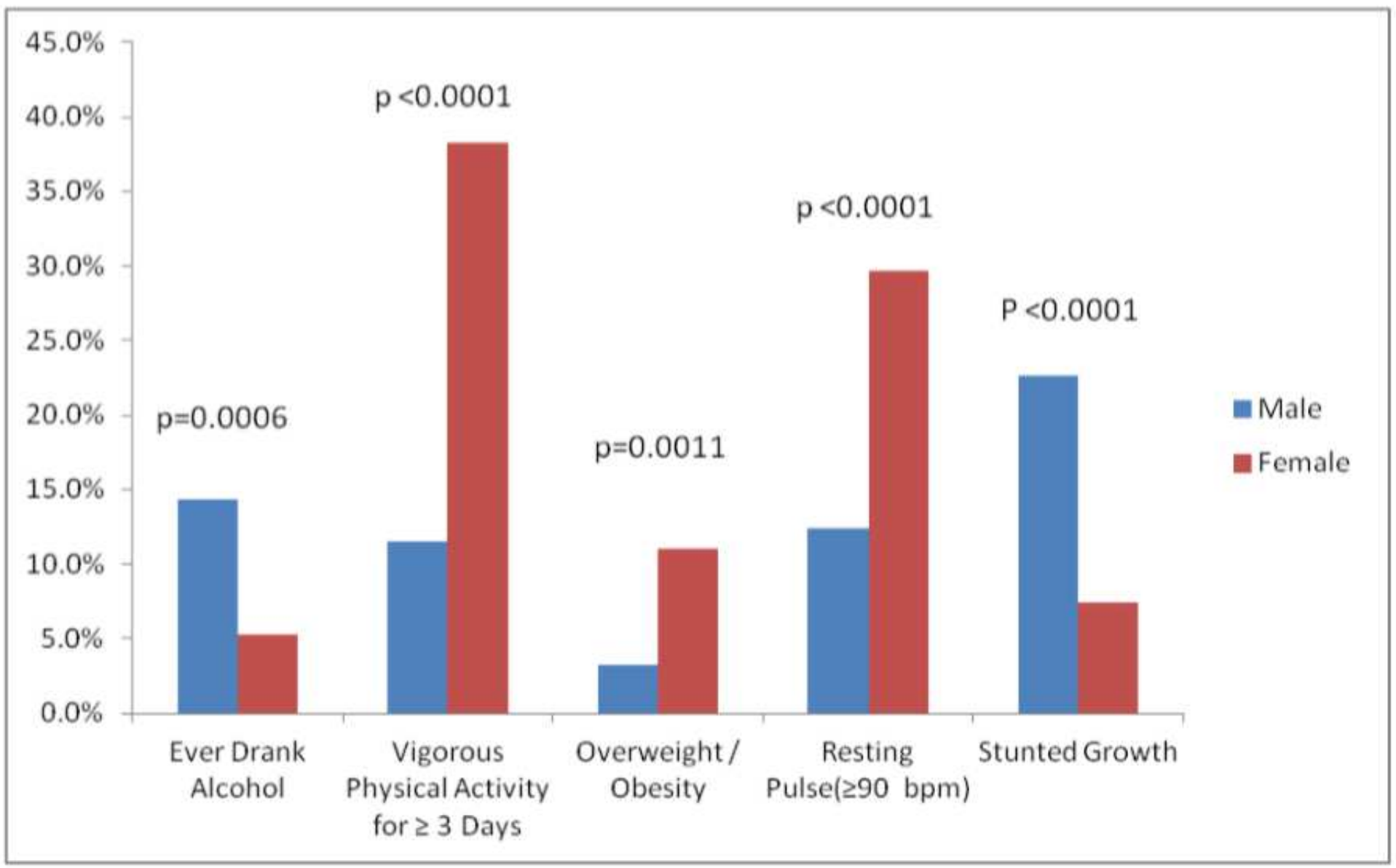

Figure 1

Bar graph showing risk factors for cardiovascular disease which were statistically significant different between males $(n=217)$ and females $(n=283)$ participating in adolescents' cohort study 


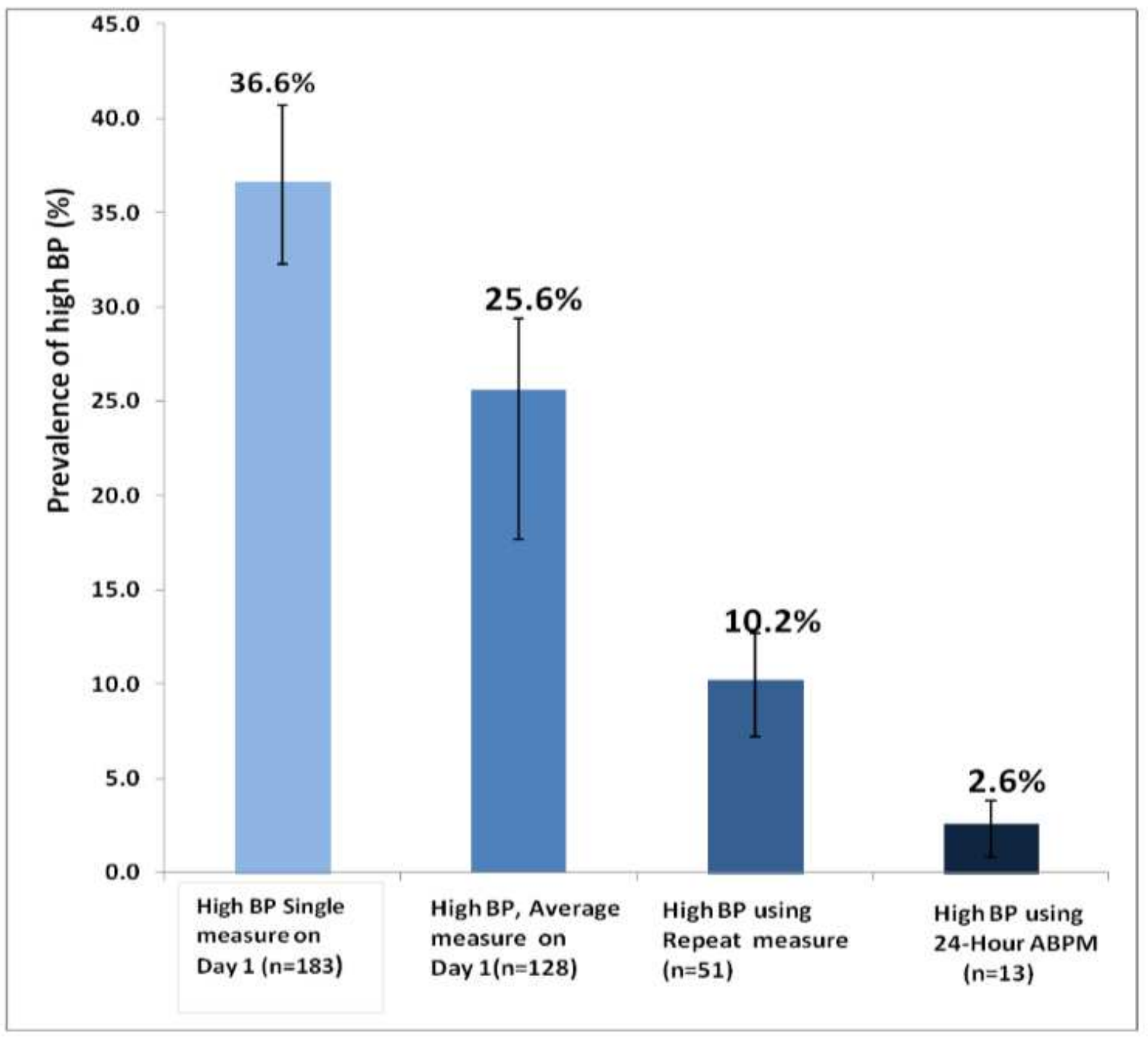

Figure 2

Prevalence of high BP with their corresponding estimation methods among adolescents enrolling in adolescents' cohort study $(\mathrm{N}=500)$.

\section{Supplementary Files}

This is a list of supplementary files associated with this preprint. Click to download.

- SupplimentaryTableFinal10.12.2020.pdf 